\title{
Optimal dosimeter deployment into a smart city IoT platform for wideband EMF exposure assessment
}

\author{
L. Diez, L. Rodríguez de Lope, R. Agüero \\ University of Cantabria \\ [ldiez, laura, ramon]@tlmat.unican.es
}

\author{
Y. Corre, J. Stéphan, M. Brau \\ Siradel \\ [ycorre, jstephan, mbrau]@siradel.com
}

\author{
S. Aerts, G. Vermeeren, L. Martens, \\ W.Joseph \\ Ghent University / iMinds, Gaston \\ [sam.aerts, gunter.vermeeren, luc.martens, \\ wout.joseph]@intec.ugent.be
}

\begin{abstract}
LEXNET project, funded by the European Commission, is introducing new concepts for the assessment of the population EMF exposure. It also aims at evaluating the capability of future technologies to reduce this EMF exposure. The platform presented in this paper is an original tool for a continuous measurement of the multi-band downlink Electric Field strength at a scale of a city, relying on the SmartSantander sensor network that was developed in a previous project. The testbed integrates a new equipment to capture the E-field strength, i.e. connected dosimeters. The work that is reported here focuses on the deployment of those dosimeters in the city of Santander. The presented methodology uses both simulation and measurement to achieve an optimal design, i.e. a dosimeter deployment that provides relevant exposure statistics and allows for the creation of exposure maps. Results on the pre-deployment simulations are given in this document, while the complete design optimization will be demonstrated later in Year 2015.
\end{abstract}

Keywords - Electromagnetic field exposure; Dosimeter; Smart city; Internet of Things; Simulation; Exposure Index; Interpolation

\section{INTRODUCTION}

Although no adverse health effects caused by wireless devices usage have been demonstrated, the exposure to electromagnetic fields (EMF) generated by radiofrequency (RF) sources is often cited as a cause of public concern [1]. On the other hand, mobile phones and other wireless devices, like tablets or laptops, have become an essential part of everybody's life, and it is not realistic promoting their usage reduction. In this sense, the "Low EMF Exposure Future Networks" (LEXNET) project, funded by the European Commission in its Seventh Framework Programme, aims at designing a set of techniques and procedures to reduce the exposure of a population in an area without jeopardizing their quality of experience.

One of the main outcomes of LEXNET is the definition of a new metric, which has been coined as Exposure Index (EI), and the methodology to determine and assess such exposure over real networks [2]. The EI has been conceived to assess the global exposure of the population, considering both the personal devices and network access elements. In the framework of LEXNET, the study presented herewith focuses on the characterization of far-field exposure of the population, at different radio-frequencies. We are interested in studying real networks and therefore this analysis is being carried out within a medium-size city.

The evaluation of the EI requires far-field and near-field assessment over large scenarios (i.e. a city district, business area) and extended periods of time. While in the past the far-field exposure has been measured by means of sophisticated devices, such as spectrum analyzers, the need for a large-scale assessment makes it necessary to explore novel measurement methodologies. In this sense, LEXNET project has opted for the deployment of low-complexity dosimeters [3] in the city of Santander (Spain), resulting in a sensor network that acts as a large-scale measurement tool. In particular, they will be integrated within an Internet of Things (IoT) platform, the SmartSantander [4] testbed, which is the main outcome of a former EU project on Smart-Cities. The low-complexity dosimeters will act as regular IoT nodes to facilitate the process of gathering the produced data offered by the testbed. In a next phase, the intention is to combine the dosimeter measurements from different locations to generate exposure maps as well as to study the potential correlation of such measurements with different parameters, such as the period of time. The knowledge that will be acquired can afterwards be exploited to fine-tune simulation platforms, which could then be used to assess the possibilities of some management techniques that are being studied within the project framework.

In order to optimize the low-complexity dosimeters deployment, it is necessary to consider the current distribution of access elements over the target area, as well as the particular characteristics (i.e. type of buildings, streets width) of such area. This paper describes the methodology that will be followed in LEXNET to select the best locations of the dosimeters to be deployed, subsequently, to ensure an optimal grid for the interpolation of the electric-field (EField) strength over the study area. It is based on a combination of simulation techniques, which consider the scenario characteristics, and an iterative procedure, which addresses the optimization of the sensor deployment. In a nutshell, based on the simulation results over the area, this procedure involves a sequential deployment that, at each 
step, makes use of an optimization algorithm, which is fed with real measurements of the previous step.

The document is structured as follows: Section II describes the target area and provides an overview of the whole deployment methodology. Section III details the simulation configuration which sets the basis for the deployment. Section IV describes the measurement procedure and presents some preliminary results obtained by means of a test deployment. The sequential algorithm is presented in Section V, highlighting the benefits of this approach. Finally, Section VI concludes the paper, pointing out the main outcome obtained by this ongoing study and the research lines that get opened as a result of this work.

\section{OVERARCHING DESCRIPTION OF THE METHODOLOGY}

The dosimeter network will be deployed in the downtown of Santander covering a surface of $0.35 \mathrm{~km}^{2}$ (highlighted in Fig. 1), which contains both open areas and narrow streets between high buildings. As it is shown in Fig. 1 , there are a large number of base stations either within the target area or nearby.

It is worth highlighting that, due to the "spectrum refarming" techniques that are being used by the operators, bands that were initially devoted to a specific technology may accommodate a different one (for instance, the use of traditional 2G bands for LTE deployment) [5].

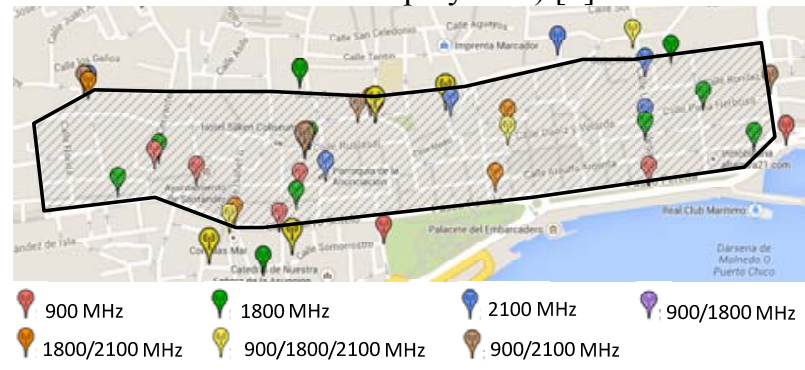

Fig. 1. Target area (striped area) and positions and technologies of the base stations deployed in the immediate vicinity.

The ultimate goal of this study is to characterize the RFEMF exposure in the area and, for that, the following two phases will be performed: first, to obtain a good approximation of the statistical distribution of the E-field strength inside the target area, and secondly, to develop an interpolation model of the E-field strength with reasonable accuracy.

To achieve the former, the required number of lowcomplexity dosimeters will be estimated by means of simulation analysis, which will be discussed in Section III. The latter state, on the other hand, can be fulfilled by means of an efficient deployment procedure, in which the location selection is based on an iterative method, which considers the outcome of the previous stages to establish the locations of additional dosimeters in a new stage.

Although the available deployment points on a real deployment are more limited (specific lamp posts and walls), compared to those used in the preliminary simulation studies (streets and other publicly accessible outdoor areas), the overall proposed procedure described below (see Fig. 2) would offer high improvements compared to naive (random) deployments:

a) Initial simulations will be performed using accurate information about the target area. This will provide valuable information (model based) that will be considered during the whole deployment process.

b) Partial deployment. In an initial phase there will not be real measurements to guide the deployment, and a preliminary simulation study will be used. In the subsequent steps, the sequential algorithm will provide insights about the best possible locations.

c) After a certain period of time, the dosimeter measurements will be analyzed, spatially and temporally. This information will be used by the algorithm to estimate the best locations for the new batch of dosimeters.

d) Steps (b) and (c) are repeated until all dosimeters are distributed.

e) Once the whole deployment is completed, the obtained measurements will be used to calibrate simulation tools and models.

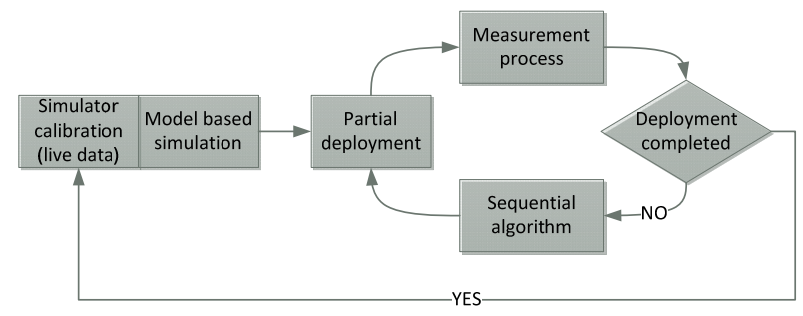

Fig. 2. Iterative deployment procedure

It is worth highlighting the different roles played by the simulation and the iterative algorithm. The former provides dimensioning insights based on statistical, mainly average, information of the electric field in the area. The iterative process, on the other hand, aims to provide a tailored deployment, since it brings about an accurate mapping of the electric field on the area.

\section{SimULATION BASED ANALYSIS OF THE EMF SENSOR DEPLOYMENT}

As can be seen in Fig. 2, radio-planning-like simulation is the first block in the overall procedure. This is a convenient way to evaluate the performance of the dosimeter network and to adjust its design before on-field deployment. The simulations do reproduce measurements, as it would be done with a dosimeter deployment, leading to an evaluation of sensing error statistics.

The basic principles of the simulation study are as follows. First, the downlink E-field strength is predicted over a map (or pixel grid) that covers the whole study area. This map is considered as a realistic EMF realization, which is used afterwards as a reference. Then, the dosimeter measurements are emulated by picking sample values in the reference map, at locations chosen according to the deployment strategy under test. Finally, the E-field strength 
statistics computed from the whole map are compared to the E-field strength statistics derived from the emulated measurements, in order to evaluate the performance of the sensor network. This study has to be conducted in each frequency band where cellular networks are operating, i.e. at $900 \mathrm{MHz}, 1800 \mathrm{MHz}$ and $2100 \mathrm{MHz}$ in Santander, considering all base-stations from four operators.

\section{A. Simulation setup}

The results reported in this paper are obtained from the four HSPA networks operating at $2100 \mathrm{MHz}$ : 169 macrocells in a surface of $0.45 \mathrm{~km}^{2}$ composed of Santander downtown plus a margin of 500 meters. The downlink Efield strength from those HSPA networks is predicted in the downtown area.

Some base station parameters (antenna pattern, orientation and height) were unknown for the study. Therefore typical values have been applied to those base stations: directional antenna with $60^{\circ}$ horizontal beamwidth, $12^{\circ}$ vertical beamwidth and maximum gain of $14 \mathrm{dBi}$, height of the support (building, pylon) plus two meters.

The downlink EMF exposure, in the sense of LEXNET, depends on the dynamic transmit power, which is below the maximum transmit power usually considered in EMF exposure regulation. This dynamic transmit power is function of the average percentage of resources allocated to serve the network users, the so-called traffic load. For the pre-deployment dosimeter planning, which is reported here, a unique $50 \%$ traffic load is assumed for all base stations. However the measurements that will come from the first deployed dosimeters will later permit a tuning of the traffic load per frequency band and operator, leading to a refined deployment simulation.

The simulation relies on the $3 \mathrm{D}$ representation of the environment, composed of the raster terrain altitude (resolution 5 meters) and 3D vectors to model buildings, vegetation, bridges and water bodies. The propagation loss is computed by the Volcano deterministic model [6] that predicts physical propagation mechanisms (diffraction in particular) to provide realistic downlink E-field strength maps. Even with a deterministic model, the shadowing loss suffers from a prediction error (when compared to real measurements) that usually follows a lognormal distribution. A standard deviation of about $7 \mathrm{~dB}$ and a correlation distance of 50 meters is supposed here, which is taken into consideration by simulation of random shadowing contributions (independent between not-colocated base stations) and the creation of several realizations. The dosimeters will be installed at a height of few meters above the streets; and the measurements will be converted (from statistical rules) to street-level or in-building estimates to get relevant data for the computation of the people exposure. For simplicity, the uncertainties that will be produced by those conversions are not considered here. The emulated dosimeters are assumed to be installed at the height of 1.5 $\mathrm{m}$. And only the error on the outdoor exposure assessment is used to evaluate the performance of the sensor networks.
Fig. 3 shows the outdoor downlink E-field strength maps predicted with a resolution of 5 meters, at one random realization, for respectively one specific network operator (Orange) and the sum of the four operators. The statistics extracted from those maps lead to the reference CDFs shown in Fig. 4.

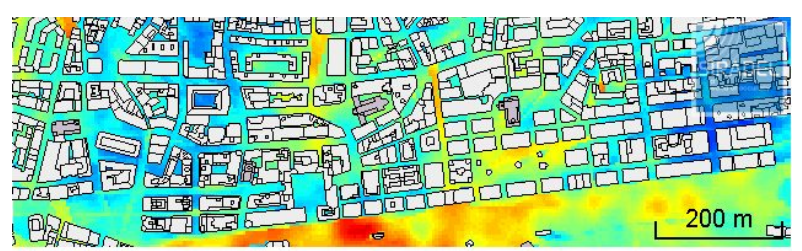

(a) HSPA network \#1

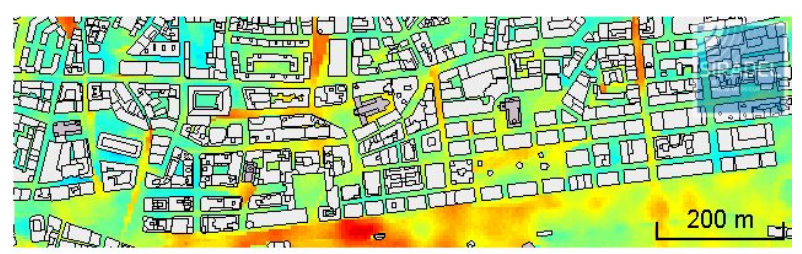

(b) All HSPA networks

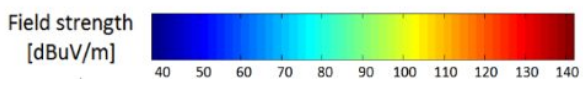

Fig. 3. Outdoor downlink E-field strength maps

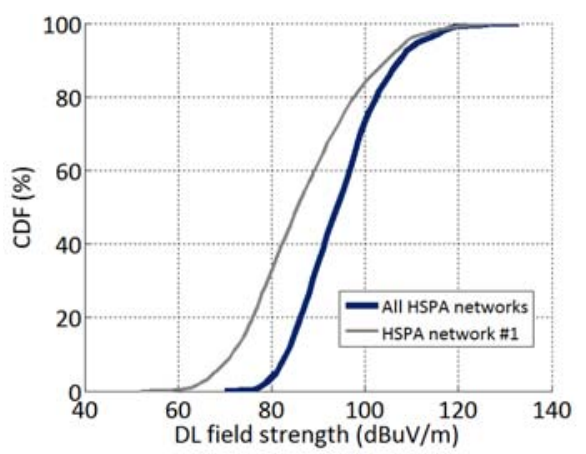

Fig. 4. CDF of the outdoor downlink E-field strength over the whole study area

These figures bring about two interesting pieces of information regarding the downlink E-field strength distribution in a dense urban area: peaks are observed, as expected in the close vicinity of base stations; but variations over the remaining of the area are quite small, except the strong differences between confined/street areas (lower levels) and large open areas (higher levels).

\section{B. Evaluation of different deployment strategies.}

Three different deployment densities are evaluated with respectively 10,25 and 50 sensors. In all cases, the dosimeters are deployed uniformly in the streets over the whole considered area. One example is illustrated in Fig. 5.

Fig. 6 gives the CDF of the sensor measured field values from the different tested densities, obtained at one random realization. The root mean square error (RMSE) and the bias 
(or mean error) are calculated for each realization and dosimeter density. The RMSE and absolute bias values are averaged over several realizations to get the average performance statistics shown in Table 1 . We observe first that the RMSE error when measuring the exposure coming from all HSPA networks together is lower than the error related to one single network. This simply results from the fact that spatial exposure variations decrease as the number of contributing networks increase (provided the base stations from different operators are not all collocated).

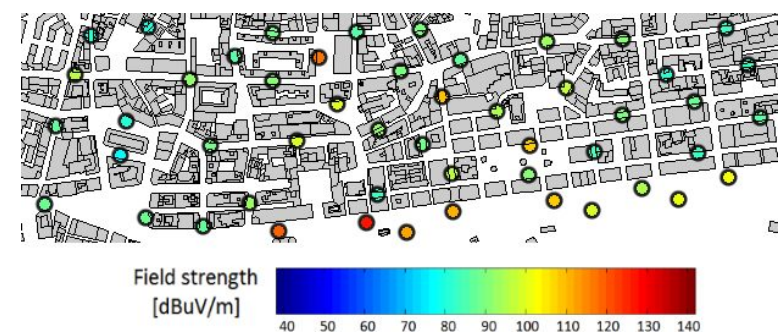

Fig. 5. Measured E-field strength levels from the 50-sensor deployment.

Besides, the most important conclusion from this study is the relationship between the number of dosimeters and the assessment error: the error degradation between 50 and 25 dosimeters is very low ( $4 \%$ on the all-networks RMSE) while it is far more significant when changing from 25 to 10 dosimeters $(47 \%)$.

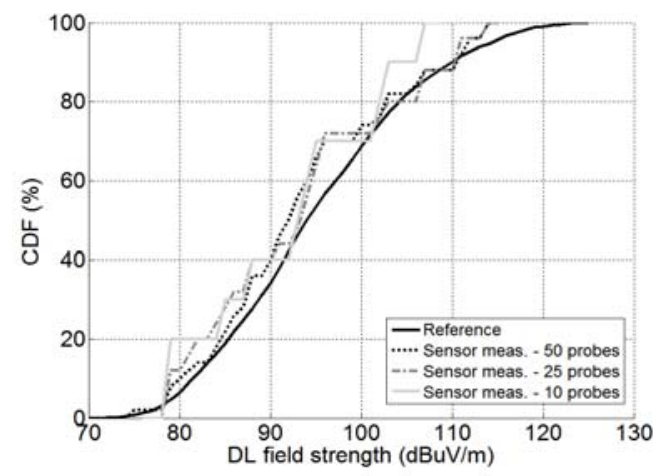

Fig. 6. E-Field strength CDF from dosimeter measurements

TABLE I. ERROR STATISTICS FROM DIFFERENT DOSIMETER DEPLOYMENTS

\begin{tabular}{|c|c|c|c|c|c|c|}
\hline \multirow{2}{*}{ Sensor network with } & \multicolumn{2}{|c|}{50 probes } & \multicolumn{2}{|c|}{25 probes } & \multicolumn{2}{|c|}{10 probes } \\
\hline & $\begin{array}{c}\text { Mean } \\
\mid \text { |bias| } \\
\text { (dB) }\end{array}$ & $\begin{array}{l}\text { RMSE } \\
\text { (dB) }\end{array}$ & $\begin{array}{l}\text { Mean } \\
\text { |bias| } \\
\text { (dB) }\end{array}$ & $\begin{array}{l}\text { RMSE } \\
\text { (dB) }\end{array}$ & $\begin{array}{l}\text { Mean } \\
\text { |bias| } \\
\text { (dB) }\end{array}$ & $\begin{array}{l}\text { RMSE } \\
\text { (dB) }\end{array}$ \\
\hline Single HSPA network & 1.17 & 3.06 & 1.03 & 3.48 & 2.38 & 5.19 \\
\hline All HSPA networ & 1.38 & 2.52 & 1.40 & 2.63 & 1.71 & 3.87 \\
\hline
\end{tabular}

This study gives obviously valuable indicators to make a trade-off between accuracy and deployment cost. Considering that the maximum number of dosimeters available for the Santander deployment is 50 , and that the dosimeters not used in the downtown area will serve for testing other areas, it looks like 25 is a reasonable trade-off that will provide an error very close to the optimal.

\section{MEASUREMENT PROCESS AND PRELIMINARY RESULTS}

The sensor network to be deployed will provide a continuous measurement of the downlink field strength $(\mathrm{mV} / \mathrm{m})$ of the four frequency bands currently in operation within the city: $900 \mathrm{MHz}, 1800 \mathrm{MHz}, 2100 \mathrm{MHz}$ and 2400 MHz. The deployed cellular technologies have been selected as being the main cause for almost all the outdoor exposure, while WiFi $2.4 \mathrm{GHz}$ band has been included to assess the exposure from outdoor WiFi hot-spots together with potential contribution from indoor WiFi sources.

Before starting the deployment process, a test installation, embracing two dosimeters, has been deployed. It has two main objectives: first, to verify the correct integration of the dosimeter as a new IoT node within the smart-city testbed; additionally, this installation brings the opportunity to start assessing the variation of the E-field strength under different circumstances. In particular one of the two dosimeters is deployed outdoors, while the second one is indoors, at a university laboratory, with a high number of WiFi access points.

Fig. 7 depicts the E-field values measured by the outdoor dosimeter during one week time. As can be seen, there is a cyclic pattern that corresponds to the time of the day, which has, as the figure shows, a remarkable impact, especially for the 900 and $2100 \mathrm{MHz}$ bands. The figure also yields that the measurements at $1800 \mathrm{MHz}$ are almost constant, and do not depend on the particular time of day. This can be attributed to the various technologies deployed at each frequency band; further information about the bands usage can be found in [5].

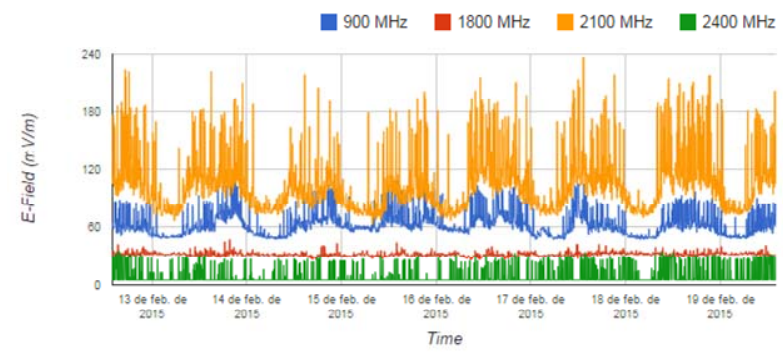

Fig. 7. Preliminary measurements outdoor

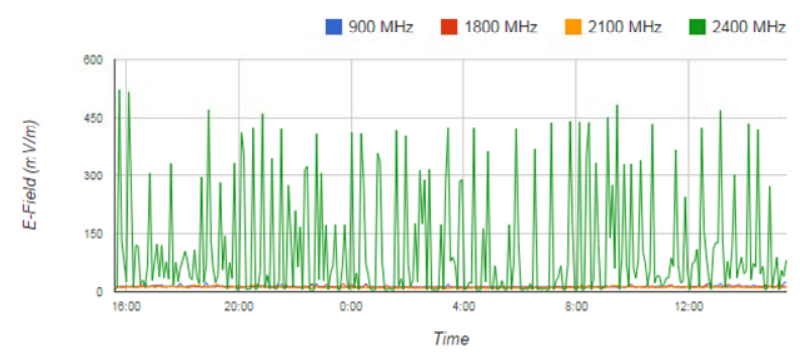

Fig. 8. Measurements indoor

Finally we have assessed the E-field strength difference that can be expected for different bands when they are measured either indoor or outdoor. By comparing Fig. 7 and Fig. 8, we can conclude that cellular technologies are the dominant exposure source outdoors, but their induced E- 
field strength can be considered negligible within indoor environments, when compared with $\mathrm{WiFi}$, at least in scenarios where the WiFi activity is relevant (as it is the case in a university laboratory). Additionally, Fig. 8 yields another conclusion: unlike some cellular technologies, WiFi causes rather constant field value regardless the time of the day.

\section{SEQUENTIAL DESIGN ALGORITHM}

Within the framework of LEXNET, the real data obtained by means of the dosimeters deployment has two main objectives: a good approximation of the statistical distribution of the E-field in the target area, and a good interpolation model of the E-field in such area. In order to fulfill the first requirement, a certain number of dosimeters deployed at random in the area might suffice, and this number can be found via the simulations. However, the second requirement makes it necessary a smart deployment to obtain reasonable interpolation model.

The methodology that will be used in the deployment procedure is based on the works [7] and [8], where a sequential measurement method was applied to an urban, outdoor RF-EMF exposure assessment campaign. The use of a sequential method allowed the experimenters to choose specific measurement locations more efficiently, based on the knowledge provided by earlier measurements.

The applied algorithm consists of two clashing criteria based on the requirements established at the start of the measurement campaign. On the one hand, it is always desirable to perform measurements in previously uncharted areas (exploration); while, on the other hand, regions which are deemed interesting (e.g., where the exposure is rapidly varying and/or very high compared to other regions) are sought to be more accurately characterized (exploitation). The algorithm gives each potential location a certain score according to the result of this trade-off between exploration and exploitation, and the highest-scoring locations then represent the next stage of deployment.

Intuitively, the main advantage of an iterative procedure is its efficiency. From a number of electric-field measurements and the following interpolation of the electric-field strength over the target area, one selects the next measurement locations based on their ability to enhance the interpolation model, by lying in areas that are either under-investigated or that show a highly varying exposure.

\section{CONCLUSION AND FUTURE PLANS}

In spite of the growing concern on the exposure to EMF induced by wireless communication technologies, a systematic methodology to assess the real EMF exposure has not yet been developed. There exist tools to measure the EMF, but their usage is limited to particular areas and time periods and they usually require a manual configuration and operation. Hence, they cannot be efficiently used to assess the overall EI over a broad area and during long periods of time. In order to overcome these limitations, this paper presents an ongoing work to deploy a large-scale measurement tool, based on an IoT testbed within a medium-size city.

We have presented the first results of an initial deployment, which verified the successful integration of the dosimeters within the smart-city testbed and the correct data acquisition process. A simulation based study of the deployment area is presented, showing that 25 dosimeters will be able to provide an appropriate accuracy for the area of interest. In addition, we have discussed a systematic methodology to establish the most suitable dosimeter deployment, based on a sequential algorithm, where locations of new batches of dosimeters are based on the analysis of the previous deployment.

Once the sensor density and the deployment methodology have been established, we will soon start with the actual installation and deployment. The corresponding testbed be exploited to fine-tune simulation platforms, which can afterwards be used to assess the management techniques that are being studied to reduce the EI. In addition, the final outcome will also allow assessing the EMF induced by real cellular networks within a mediumsized city.

\section{ACKNOWLEDGMENT}

This paper reports work undertaken in the context of the project LEXNET. LEXNET is a project supported by the European Commission in the 7th Framework Programme (GA n 318273 ). For further information, please visit www.lexnet-project.eu. In particular, we are grateful to the partners from SATIMO who collaborate in the Santander testbed development via the design and production of the dosimeters.

\section{REFERENCES}

[1] "Eurobaromenter 73.3, Electromagnetic Fields. June 2010." [Online]. http://ec.europa.eu/public_opinion/archives/ebs/ebs_347_en.pdf

[2] M. Tesanovic et al., "The LEXNET project. wireless networks and emf: Paving the way for low-emf networks," Vehicular Technology Magazine, IEEE, vol. 6, no. 2, June 2014.

[3] L. Diez et al., "Design and integration of a low-complexity dosimeter into the smart city for EMF assessment", Networks and Communications (EuCNC), 2014 European Conference on, pp.1-5, June 2014. DOI: 10.1109/EuCNC.2014.6882637.

[4] "EU FP7 Project Smart Santander (ID 257992)." [Online]. http://www.smartsantander.eu

[5] T. Sarrebourse et al., "Towards EMF exposure assessment over realcellular networks: an experimental study based on complementary tools". International Symposium on Wireless Communicaton Systems (ISWCS), August 2014.

[6] Y. Corre and Y. Lostanlen, "Three-dimensional Urban EM Wave Propagation Model for Radio Network Planning and Optimization Over Large Areas", IEEE Trans. on Vehicular Technology, Oct. 2009.

[7] S. Aerts et al., "Exposure assessment of mobile phone base station radiation in an outdoor environment using sequential surrogate modeling". Bioelectromagnetics 34, 300-311.

[8] S. Aerts et al., "Assessment of outdoor radiofrequency electromagnetic field exposure through hotspot localization using kriging-based sequential sampling". Environ. Res. 126, 184-191. 